\title{
PRODUCCIÓN DE MACA (Lepidium meyenii Walp.) EN CONDICIONES DE INVERNADERO USANDO GUANO FERMENTADO Y PGPRS BAJO DOS SISTEMAS DE CULTIVO EN SAN PEDRO DE CAJAS - JUNÍN, PERÚ
}

\section{YIELD OF MACA (Lepidium meyenii Walp.) IN GREENHOUSE CONDITIONS USING FERMENTED DUNG AND PGPRS UNDER TWO CULTURE SYSTEMS IN SAN PEDRO DE CAJAS - JUNIN, PERU}

\author{
Abelardo Calderón Rodríguez ${ }^{1}$ y Cesar Cáceres Cunto ${ }^{2}$
}

\begin{abstract}
Resumen
El cultivo de la maca Lepidium meyenii Walp. se ha expandido considerablemente, debido a la gran demanda del producto en el extranjero y ha llegado incluso a ocupar terrenos de pastizales. Por otro lado, los rendimientos obtenidos por metro cuadrado con los métodos de cultivo tradicionales son bajos. En la presente investigación, se evalúa el rendimiento de la maca en función del peso fresco de los hipocótilos al momento de la cosecha como respuesta a la implementación de un nuevo paquete tecnológico compuesto por un invernadero con condiciones semicontroladas, trasplante de plántulas, riego por goteo, guano de alpaca fermentado, aplicación de rhizobacterias promotoras del crecimiento vegetal (PGPRs) a los tratamientos y dos sistemas de cultivo, el vertical y el horizontal. Se demuestra estadísticamente que el sistema horizontal supera significativamente al sistema vertical y además, el tratamiento "suelo virgen más guano fermentado" da mejores resultados que los otros tratamientos. La aplicación de los PGPRs a los tratamientos no tuvo ningún efecto positivo en el cultivo. Los datos micrometeorológicos registrados al interior del invernadero nos muestran que las condiciones de intensidad luminosa, temperaturas diurnas y nocturnas y concentración de $\mathrm{CO}_{2}$ fueron favorables para la maca (planta C3). Para concluir, el rendimiento de los hipocótilos frescos de maca con el nuevo sistema es 3.5 a 4 veces mayor que el método tradicional, asimismo el ciclo vegetativo se acorta y podemos cultivas dos campañas al año.
\end{abstract}

Palabras clave: invernadero, producción, maca, hipocótilo y PGPR.

\begin{abstract}
The maca, Lepidium meyenii Walp. culture has been expanded to wild and grassland soils in highland Peruvian central sites, owing to the great demand of the product and to the low productivity per square meter with the traditional methods of culture. In the present work we value the yield of the maca in terms of fresh weigh of hypocotyls as a response to a new technologic package composed by a semicontroled greenhouse ,transplant of maca plantlets, drop watering, alpaca's fermented dung, PGPR application to the treatments and two culture systems; the vertical one and the horizontal. Statistically, the horizontal system is better than the vertical and besides, the treatment "virgin soil plus alpaca's fermented dung" gives the better results than other treatments. The application of the plant growth-promoting rhizobacteria (PGPR) on the treatments do not had positive effects on yields. According to the micrometeorological data registered on the greenhouse, the conditions of light intensity, day and night temperature and $\mathrm{CO}_{2}$ concentration were benign for the maca (C3 plant) inside the greenhouse. To conclude, the yield of fresh maca hypocotyls with this new system is 3.5 to 4 times more than the traditional method and besides the vegetative cycle shortens so we can cultivate twice a year.
\end{abstract}

Key words: greenhouse, yield, maca, hypocotyls, PGPR.

\section{Introducción.}

La maca (Lepidium meyenii Walp.) es una de las pocas especies vegetales que se cultivan en las zonas altoandinas, entre los 3700 y $4500 \mathrm{msnm}$ y tiene un gran valor alimenticio, medicinal y genético como fuente de genes de resistencia y adaptabilidad a las bajas temperaturas, altas irradiancias, incluso radiación ultravioleta, déficit hídrico, entre otros factores adversos. (King, 1988; Garay, 1992; Quiros et al., 1996; Obregón, 1998). En los últimos años ha cobrado gran importancia debido a que se ha conocido con más detalle la bioquímica de sus metabolitos secundarios y la actividad de estos sobre la alimentación y la salud humana (Dini et al., 1994; Marthe et al., 2003). 
Se ha determinado las concentraciones de macamidas, macaenos, glucosinolatos, flavonoides, ácidos grasos, entre otros, tanto en hipocótilos en estado fresco como en hipocótilos secos (Dini et al., 1994; Quiros et al., 1996). La procedencia del material vegetal es determinante en la composición o calidad bioquímica del producto. Así, la combinación de los factores ambientales en el lugar del cultivo regulará la expresión genética del metabolismo y la producción de los diferentes metabolitos (Moreno, 1970; Moreno, 1985; Calderón, 1988; Heller et al., 2000; Fageria et al., 2006).

Aparentemente la maca ocupó mayores áreas de cultivo en el pasado (Johns, 1981). En 1992, el cultivo estaba restringido a las localidades ubicadas en la Meseta del Bombón (Huayre, Vico, Uco, Carhuamayo, Ninacaca, etc.) pero debido a la demanda creciente el cultivo se ha extendido a otros lugares como Ancash, Huánuco, Ayacucho, Puno, Junín. (Aliaga, 1999). Entre los años 2014 al 2016 se verificó la mayor extensión sembrada debido principalmente a la demanda del mercado Chino, según lo expuesto por Alcántara (2015), esta demanda tuvo como consecuencias el tráfico de tierras en la región, para lograr un incremento en la producción que cubra los requerimientos. Esto se evidenció en la apertura de nuevas áreas de cultivo en campos que eran destinados a la ganadería o de pastos naturales, más que en el incremento del rendimiento por unidad de área sembrada. Para el año 2017 ha ocurrido un descenso significativo de la demanda y como consecuencia los precios han caído, por lo tanto, se espera que la extensión de siembra para el siguiente año también se reduzca (La República, 2017). Por otro lado, no hay muchos estudios en relación a la fisiología del cultivo $\mathrm{y}$ este se sigue haciendo en forma empírica, como lo hacían nuestros antepasados y por tanto, los rendimientos de hipocótilos (Strasburger et al. 1986) frescos son bajos, variando entre $280 \mathrm{~g} \mathrm{PF} / \mathrm{m}^{2}$ a $410 \mathrm{~g}$ $\mathrm{PF} / \mathrm{m}^{2}$ y $99.9 \mathrm{~g}$ PS a $149.7 \mathrm{~g} \mathrm{PS} / \mathrm{m}^{2}$ (Tello et al., 1992).

La maca tolera temperaturas muy bajas, de $-7{ }^{\circ} \mathrm{C}$ a $-10{ }^{\circ} \mathrm{C}$ durante las noches y madrugadas, y de $15^{\circ} \mathrm{C}$ a $22{ }^{\circ} \mathrm{C}$, al medio día. Las lluvias irregulares, la alta irradiación que reciben los campos en la puna en determinados días, los fuertes vientos y la escasa materia orgánica de los suelos son algunos de los factores que limitan el crecimiento y la producción del cultivo.

Otro factor responsable de los bajos rendimientos es la práctica tradicional de siembra. Esta consiste en acondicionar el suelo, agregándole materia orgánica y algo de ceniza. Es preferente que este suelo sea virgen o haya descansado por más de cinco años (Tapia, 1991; Obregón, 1998). La siembra de la semilla botánica se hace al voleo en las primeras horas de la mañana, luego la semilla es cubierta ligeramente con ramas o con la ayuda del pisoteo de las ovejas y se espera que la lluvia haga el resto del trabajo. Por esta razón, la siembra debe hacerse en cuanto se avizoren las primeras lluvias las cuales en estos últimos años han demorado en presentarse debido al cambio climático. La siembra al voleo resulta en una alta desuniformidad del cultivo Hay zonas en el campo con alta densidad de plantas y otras con pocas o muy dispersas, en promedio el índice de área foliar es muy bajo.

Por otro lado, las bajas temperaturas limitan la actividad microbiana de descomposición de la materia orgánica y el abastecimiento de minerales y otros compuestos activos a las plantas. La aplicación de algunas Rhizobacterias Promotoras del Crecimiento Vegetal (PGPRs) podría ser beneficiosas en el incremento del crecimiento de las plantas y en su tolerancia a otros microorganismos patógenos. Estos microorganismos pueden estimular la germinación y el enraizamiento; incrementar la disponibilidad y suministro de nutrientes; mejorar la estructura del suelo a través de la formación de agregados estables y proteger a la planta de estreses bióticos y abióticos, dándole mayor resistencia o tolerancia (Calvo, 2008).

La presente investigación fue planteada con el objetivo de evaluar la producción de hipocótilos en un sistema de cultivo en columnas (vertical) versus un sistema horizontal en respuesta a la aplicación de guano fermentado e inoculación de PGPRs. bajo condiciones semicontroladas de invernadero. Se realizó el trasplante y riego por goteo en ambos sistemas de cultivo.

\section{Materiales y métodos. \\ Área y Condiciones Ambientales del Estudio.}

La presente investigación se realizó en una ladera de la comunidad San Pedro de Cajas ubicada a 4210 msnm, latitud $11^{\circ} 15^{\prime} 04^{\prime \prime} \mathrm{S}$ y longitud $75^{\circ} 51^{\prime} 42^{\prime \prime} \mathrm{W}$, provincia de Tarma, Departamento Junín.

Las temperaturas medias diurnas y nocturnas promedio en campo abierto durante la época de cultivo fueron $9.72{ }^{\circ} \mathrm{C} \mathrm{y} 4.56{ }^{\circ} \mathrm{C}$ respectivamente, mientras que al interior del invernadero se registraron $22.5^{\circ} \mathrm{C}$ y 7.92 ${ }^{\circ} \mathrm{C}$. (Tabla 1)

Se construyó un invernadero de $400 \mathrm{~m} 2$, de doble capilla, con dos naves yuxtapuestas longitudinalmente y con ventilación cenital en cada una de las cumbreras. Las caras laterales se cerraron con malla antiáfida y plástico agrícola a manera de cortina para subirla o bajarla según sea la necesidad de controlar el ambiente interior.

Se instaló un sistema de riego por goteo para cada uno de los tratamientos a partir de un tanque elevado y con agua proveniente de un puquial cercano recubierto con geomanta. Para evitar el efecto de los fuertes vientos se tuvo que construir barreras rompe vientos de $4 \mathrm{~m}$ de altura con malla arpillera a ambos lados. Para evitar el daño por el ganado se cercó el contorno del invernadero con postes y alambre de púas. 
Tabla 1. Promedio de temperaturas al interior $y$ exterior del invernadero durante el periodo del cultivo en San Pedro de Cajas - Junín.

\begin{tabular}{|c|c|c|c|c|}
\hline MESES & \multicolumn{2}{|c|}{$\begin{array}{l}\text { TEMPERATURA } \\
\text { INTERNA }{ }^{\circ} \mathrm{C}\end{array}$} & \multicolumn{2}{|c|}{$\begin{array}{l}\text { TEMPERATURA } \\
\text { EXTERNA }{ }^{\circ} \mathrm{C}\end{array}$} \\
\hline dic-14 & $\begin{array}{l}\text { máxima } \\
\text { mínima } \\
\text { diurna } \\
\text { nocturna }\end{array}$ & $\begin{array}{r}33.03 \\
0.52 \\
24.9 \\
8.65\end{array}$ & $\begin{array}{l}\text { máxima } \\
\text { mínima } \\
\text { diurna } \\
\text { nocturna }\end{array}$ & $\begin{array}{r}12.39 \\
2.74 \\
9.97 \\
5.15\end{array}$ \\
\hline ene-15 & $\begin{array}{l}\text { máxima } \\
\text { mínima } \\
\text { diurna } \\
\text { nocturna }\end{array}$ & $\begin{array}{r}28.55 \\
1.46 \\
21.77 \\
7.29\end{array}$ & $\begin{array}{l}\text { máxima } \\
\text { mínima } \\
\text { diurna } \\
\text { nocturna }\end{array}$ & $\begin{array}{r}15.05 \\
2.25 \\
9.6 \\
4.7\end{array}$ \\
\hline feb-15 & $\begin{array}{l}\text { máxima } \\
\text { mínima } \\
\text { diurna } \\
\text { nocturna }\end{array}$ & $\begin{array}{r}29.96 \\
1.73 \\
22.9 \\
8.79\end{array}$ & $\begin{array}{l}\text { máxima } \\
\text { mínima } \\
\text { diurna } \\
\text { nocturna }\end{array}$ & $\begin{array}{l}11.8 \\
3.21 \\
9.67 \\
5.34\end{array}$ \\
\hline mar-15 & $\begin{array}{l}\text { máxima } \\
\text { mínima } \\
\text { diurna } \\
\text { nocturna }\end{array}$ & $\begin{array}{r}27.48 \\
1.05 \\
20.87 \\
7.65\end{array}$ & $\begin{array}{l}\text { máxima } \\
\text { mínima } \\
\text { diurna } \\
\text { nocturna }\end{array}$ & $\begin{array}{r}12.36 \\
1.12 \\
9.55 \\
3.93\end{array}$ \\
\hline abr-15 & $\begin{array}{l}\text { máxima } \\
\text { mínima } \\
\text { diurna } \\
\text { nocturna }\end{array}$ & $\begin{array}{r}29.53 \\
1.08 \\
20.87 \\
7.65\end{array}$ & $\begin{array}{l}\text { máxima } \\
\text { mínima } \\
\text { diurna } \\
\text { nocturna }\end{array}$ & $\begin{array}{c}12.5 \\
1.38 \\
9.72 \\
4.16\end{array}$ \\
\hline may-15 & $\begin{array}{l}\text { máxima } \\
\text { mínima } \\
\text { diurna } \\
\text { nocturna }\end{array}$ & $\begin{array}{r}29.68 \\
-0.60 \\
22.11 \\
6.97\end{array}$ & $\begin{array}{l}\text { máxima } \\
\text { mínima } \\
\text { diurna } \\
\text { nocturna }\end{array}$ & $\begin{array}{l}12.8 \\
1.03 \\
9.86 \\
3.97\end{array}$ \\
\hline Total campaña & $\begin{array}{l}\text { máxima } \\
\text { mínima } \\
\text { diurna } \\
\text { nocturna }\end{array}$ & $\begin{array}{r}29.7 \\
0.87 \\
22.5 \\
7.92\end{array}$ & $\begin{array}{l}\text { máxima } \\
\text { mínima } \\
\text { diurna } \\
\text { nocturna }\end{array}$ & $\begin{array}{c}12.3 \\
1.58 \\
9.72 \\
4.56\end{array}$ \\
\hline
\end{tabular}

Instalación del Experimento.

El invernadero fue dividido en dos mitades longitudinales, en la primera mitad se instaló el sistema de cultivo vertical (SCV) con 72 columnas dispuestas en cuatro hileras con 18 columnas cada una. Cada columna se construyó con ocho macetas cuadradas de tecnopor de 2.61 de capacidad para cada una, superpuestas y dejando libre las cuatro esquinas de modo que cada columna tuvo 32 plantas. En la otra mitad, se instaló el sistema de cultivo horizontal ( $\mathrm{SCH})$ con 36 parcelas dispuestas en cuatro bloques de nueve parcelas cada una. Cada parcela fue de $0.6 \mathrm{~m} \mathrm{x} 4 \mathrm{~m} \mathrm{x}$ $0.15 \mathrm{~m}$. Además, se tuvo que retirar la tierra de chacra usada para reemplazarla por el mismo volumen de suelo virgen. El número de repeticiones fue de cuatro (Figura 1).

Los substratos: suelo virgen (S.V.); suelo virgen + guano fermentado (G.F.) y suelo virgen + guano de isla (G.I.) se formularon en base al requerimiento de nitrógeno de la maca (Obregón, 1998) y se prepararon los volúmenes para los distintos tratamientos (Tabla 2).

Los PGPRs para la presente investigación fueron cepas de Bacillus sp. cepa BA8 y Pseudomonas sp. cepa PS42, aisladas en otro experimento de cultivo de maca en la misma zona, y proporcionadas por el laboratorio Marino Tabusso de la Universidad Nacional Agraria la Molina.

Las plántulas para el trasplante se obtuvieron en un sustrato de germinación constituido por musgo molido y perlita. Se llenaron 72 bandejas de 200 celdas cada una. La primera inoculación con los PGPR se hizo remojando la semilla durante media hora. El trasplante a los lugares definitivos (tratamientos) se hizo a los 52 días después de la siembra e inmediatamente se realizó la segunda inoculación en los tratamientos correspondientes. La tercera inoculación se realizó a los 147 días después de la siembra.

En el sistema de cultivo vertical se sembraron en promedio 26.6 plantas $/ \mathrm{m}^{2}$, mientras que en el sistema de cultivo horizontal, se colocaron 100 plantas $/ \mathrm{m}^{2}$. El manejo del microclima al interior del invernadero, especialmente al medio día, fue fundamental para el adecuado desarrollo de las plantas. Este básicamente consistió en abrir o cerrar las cortinas, según los cambios de temperatura, además de humedecer el interior del invernadero por aspersión. La microestación meteorológica instalada al interior del invernadero nos sirvió para este propósito. Ver anexos $1 \mathrm{a}, 1 \mathrm{~b}$ y $1 \mathrm{c}$.

Evaluaciones y Diseño Experimental.

Se hizo un monitoreo del microclima al interior y fuera del invernadero instalando una microestación meteorológica. Se registraron las temperaturas, la iluminación, la humedad relativa, las concentraciones de $\mathrm{CO}_{2}$, entre otros.
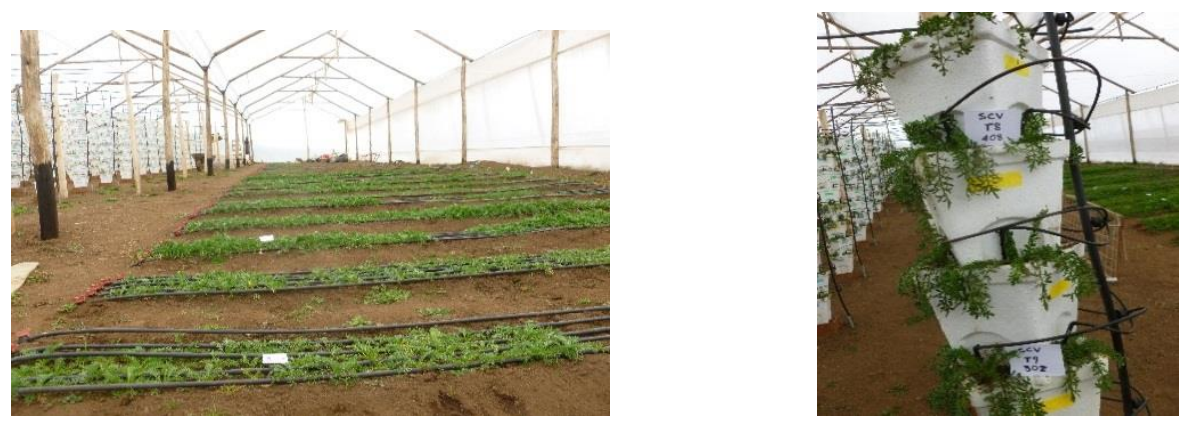

Figura 1. A la izquierda: sistema de cultivo horizontal con riego por goteo. A la derecha: sistema vertical con riego por goteo. 
Tabla 2. Composición de substratos para los Tratamientos.

\begin{tabular}{|c|c|c|c|c|}
\hline $\begin{array}{l}\text { SISTEMA DE } \\
\text { CULTIVO }\end{array}$ & $\begin{array}{r}\text { SUELO } \\
\text { VIRGEN }\end{array}$ & $\begin{array}{r}\text { GUANO } \\
\text { FERMENTADO }\end{array}$ & $\begin{array}{l}\text { GUANO } \\
\text { DE ISLA }\end{array}$ & TOTAL \\
\hline SCV & 584.21 & 195.81 & & 7801 \\
\hline MACETAS & 769.51 & ............ & 10.51 & 7801 \\
\hline $\begin{array}{l}\text { Efectivo } \\
2.61 \mathrm{c} / \text { maceta. }\end{array}$ & 780.01 & ……... & ......... & 7801 \\
\hline $\mathrm{SCH}$ & 4812.61 & 587.41 & & 54001 \\
\hline SUBPARCELAS & 5368.51 & ........... & 31.51 & 54001 \\
\hline $\begin{array}{l}\text { Vol. Efectivo } \\
0.36 \mathrm{~m} 3 \mathrm{c} / \mathrm{s} \text {. parcela }\end{array}$ & 5400.01 & .............. & ........ & 54001 \\
\hline
\end{tabular}

Por otro lado, se registraron los parámetros biométricos del crecimiento como son: peso fresco (PF) y peso seco de hojas (PS), raíces, hipocótilos; se midieron los diámetros polares y ecuatoriales de los hipocótilos; se tomaron los diámetros de las rosetas de hojas y se contaron las hojas.

El diseño experimental que se aplicó a ambos sistemas de cultivo fue el Bloque Completo al Azar con Arreglo Factorial 3x3 (Calzada, 1970). Se usó semilla botánica pura con $92 \%$ de germinación.

\section{TIPO DE SUSTRATO: \\ a1 $=$ Suelo Virgen (S.V.) \\ a2 = Suelo Virgen + Guano de Isla (G.I.) \\ $\mathrm{a} 3=$ Suelo Virgen + Guano Fermentado (G.F.) \\ TIPO DE INÓCULO PGPR: \\ b1= Inóculo 0. \\ b2= Inóculo 1. Pseudomona sp. cepa PS42. \\ b3= Inóculo 2. Bacillus sp. cepa BA8.}

$$
\begin{aligned}
& \text { TRATAMIENTO: } \\
& \text { T1 }=\mathrm{a} 1 \mathrm{~b} 1 \mathrm{~T} 4=\mathrm{a} 2 \mathrm{~b} 1 \mathrm{~T} 7=\mathrm{a} 3 \mathrm{~b} 1 \\
& \mathrm{~T} 2=\mathrm{a} 1 \mathrm{~b} 2 \mathrm{~T} 5=\mathrm{a} 2 \mathrm{~b} 2 \mathrm{~T} 8=\mathrm{a} 3 \mathrm{~b} 2 \\
& \mathrm{~T} 3=\mathrm{a} 1 \mathrm{~b} 3 \mathrm{~T} 6=\mathrm{a} 2 \mathrm{~b} 3 \mathrm{~T} 9=\mathrm{a} 3 \mathrm{~b} 3
\end{aligned}
$$

\section{Resultados y discusión.}

En la Tabla 1, el registro de las temperaturas al interior del invernadero, indica una máxima de $29.7^{\circ} \mathrm{C}$ y una mínima de $0.87^{\circ} \mathrm{C}$ así como temperaturas medias diurnas de $22.5{ }^{\circ} \mathrm{C}$ y nocturnas de $7.92{ }^{\circ} \mathrm{C}$. Se aprecia que el microclima al interior del invernadero es mucho más benigno que a campo abierto, salvo la máxima que se dio alrededor del mediodía y que fue controlada al abrir todas las ventanas y humedecer el entorno de los cultivos. La iluminancia incidente al interior del invernadero disminuyó entre un $12 \%$ a $20 \%$ según la hora del día con relación a la iluminación que se recibe fuera (Anexos 1a, 1b y 1c). En lo que respecta a las concentraciones de $\mathrm{CO}_{2}$ en el interior hubo de $5 \%$ a $12 \%$ más, según los meses y las horas registradas. La humedad del aire fue mayor por las mañanas $(50 \%)$ y menor al medio día (30\%), además de una ligera superioridad en el interior entre $1 \%$ a $6 \%$.

La maca es una planta $\mathrm{C} 3$ que requiere una temperatura aproximada entre $15^{\circ} \mathrm{C}$ a $25^{\circ} \mathrm{C}$ y una iluminación entre 10 klux a 40 klux como máximo para una fotosíntesis óptima (Black C, 1973; Salisbury \& Ross, 1994; Taiz \& Zeiger, 2011). Dichas condiciones se logran en los días nublados, mas no en los días soleados a campo abierto. Sin embargo, bajo la cubierta provista por el toldo del invernadero, las condiciones son más adecuadas para tal proceso (Anexos 1a, 1b y 1c).

Si bien se realizó el análisis estadístico con todos los registros de los parámetros biométricos, el presentado en este trabajo se refiere solo al promedio de peso fresco de hipocótilos, por representar con mayor exactitud la producción de campo.

El análisis estadístico para el sistema vertical, en términos de promedio de peso fresco de hipocótilos para los inóculos y los substratos respectivamente, muestra que no existen diferencias significativas entre los efectos de los inóculos 1 y 2 y el suelo sin inoculación, pero sí hay diferencias significativas entre los tres tipos de substratos. La comparación de medias se hizo con la prueba de Duncan al $0.05 \%$ de probabilidad (Tablas 3 y 4 ).

Tabla 3. Promedios de peso fresco de hipocótilos para los inóculos en el sistema vertical, y comparación de Medias Duncan al 0.05 de probabilidad.

\begin{tabular}{rlrl}
\hline B & INÓCULO & PROMEDIO & \\
\hline b3 & Microorganismo 2 & 7.784 & $\mathrm{~A}$ \\
b2 & Microorganismo 1 & 7.667 & $\mathrm{~A}$ \\
b1 & Sin microorganismo & 7.566 & $\mathrm{~A}$ \\
\hline
\end{tabular}

Tabla 4. Promedios de peso fresco de hipocótilos para los substratos en el sistema vertical, y comparaciones de Medias Duncan al 0.05 de probabilidad.

\begin{tabular}{clrl}
\hline A & SUBSTRATO & PROMEDIO & \\
\hline a3 & Suelo virgen + guano fermentado & 14.513 & A \\
a2 & Suelo virgen + guano de isla & 5.611 & B \\
a1 & Suelo virgen & 2.893 & C \\
\hline
\end{tabular}

Para el sistema horizontal, igualmente se analizaron los efectos de los inóculos y de los substratos en el peso fresco de los hipocótilos producidos, se puede observar claramente que el suelo sin inóculo no se diferencia significativamente de los suelos inoculados con Bacillus sp. o Pseudomonas sp., pero sí hay diferencias entre estos últimos (Tabla 5).

En lo que respecta al efecto de los substratos en el promedio de peso fresco de los hipocótilos, sí hay 
diferencias significativas entre los tres tipos de substratos (Tabla 6).

Tabla 5. Promedios de peso fresco de hipocótilos para los inóculos en el sistema horizontal, y comparaciones de Medias Duncan al 0.05 de probabilidad.

\begin{tabular}{clrc}
\hline B & INÓCULO & PROMEDIO & \\
\hline b3 & Microorganismo 2 & 15.743 & A \\
b1 & Sin microorganismo & 13.843 & A B \\
b2 & Microorganismo 1 & 12.297 & B \\
\hline
\end{tabular}

Tabla 6. Promedios de peso fresco de hipocótilos para los substratos en el sistema horizontal, y comparaciones de Medias Duncan al 0.05 de probabilidad.

\begin{tabular}{clrl}
\hline A & SUBSTRATO & PROMEDIO & \\
\hline a3 & Suelo virgen + guano fermentado & 19.217 & A \\
a2 & Suelo virgen + guano de isla & 13.239 & B \\
a1 & Suelo virgen & 9.427 & C \\
\hline
\end{tabular}

En la Tabla 7, correspondiente al análisis de promedios de pesos frescos de hipocótilos para los tratamientos en el sistema vertical, se observa que los tratamientos destacados y sin diferencias significativas entre ellos son T7, T8 y T9, los cuales llevan guano de camélido fermentado como abono orgánico para el suelo virgen. Se puede apreciar que la producción con el guano fermentado es 2.6 veces mayor que con el guano de isla y cinco veces más con relación al suelo virgen con o sin microorganismos.

Este mismo tipo de análisis comparativo para el sistema horizontal (Tabla 8) muestra los tratamientos sobresalientes (T9, T7 y T8). Si bien hay diferencias significativas entre T9 y T8, no se puede decir lo mismo de $\mathrm{T} 7$ que no se diferencia de T9. La relación de producción del suelo virgen + guano fermentado versus el suelo virgen + guano de isla es de 1.45 y frente al suelo virgen, es de 2.04 veces más.

Si se compara la producción entre ambos sistemas de cultivo, se puede decir que el sistema horizontal tiene los mejores promedios de pesos frescos de hipocótilos. En la Tabla 9 se muestra el análisis de varianza para peso fresco de hipocótilos en ambos sistemas de cultivo. Estos resultados pueden explicarse debido a que en el sistema horizontal, las plantas han estado expuestas a una iluminación más uniforme durante todo el día. En cambio, en el sistema vertical, las plantas de las zonas bajas han recibido menos luz que aquellas de las zonas altas. El crecimiento de las raíces en las macetas ha sido limitado, mientras que en las parcelas, estas se han extendido y profundizado más. La capacidad de retención del agua en las macetas no es buena y esta se distribuye más irregularmente, en cambio, las parcelas se riegan mejor a pesar de usar el mismo sistema de riego (goteo).

Si se compara la producción de hipocótilos frescos (maca), de acuerdo con el número de plantas que se sembraron por metro cuadrado, se tendría que en el sistema vertical, el mejor tratamiento (T7) produjo $14.729 \mathrm{~g} \mathrm{x} 26.67$ plantas, un aproximado de $392.5 \mathrm{~g}$ de maca fresca $/ \mathrm{m}^{2}$.

En el sistema horizontal, el tratamiento destacado fue el T9 con un promedio de $22.563 \mathrm{~g} /$ planta, si se hubieran plantado 100 plantas por metro cuadrado, la producción habría sido de $2256.3 \mathrm{~g}$ de $\mathrm{maca} / \mathrm{m}^{2}$. En el sistema tradicional (Tello et al., 1992), se obtuvo entre 500 a $750 \mathrm{~g}$ de maca fresca por metro cuadrado. Por lo tanto, con el sistema vertical no se logra una mejora productiva. En cambio, con el sistema horizontal y el nuevo paquete tecnológico que incluye invernadero, trasplante, riego por goteo y guano fermentado, se logra producir de 3 a 4.5 veces más. Si esto se compara con los resultados obtenidos en Junín por Tello et al. (1992), se estaría hablando de un incremento de 5.64 a 8.45 veces más, según el nivel de tecnología tradicional. Además, este sistema permite adelantar la campaña al año por cuanto se puede regular el microclima, el riego, el almácigo y se acorta el ciclo vital del cultivo en aproximadamente un mes.

\section{Conclusiones.}

De acuerdo con el análisis estadístico realizado, el sistema horizontal de cultivo superó significativamente al sistema vertical. De los sustratos utilizados, el suelo virgen, más guano fermentado, es el que destacó y ningún inóculo PGPR usado en el experimento tuvo efecto positivo. Con el nuevo paquete tecnológico, probado en las condiciones de San Pedro de Cajas, se obtiene de 3 a 4.5 veces más rendimiento que con los sistemas tradicionales y a la vez el período vegetativo se acorta en aproximadamente un mes.

Tabla 7. Promedio de peso fresco de hipocótilos en los Tratamientos (T) y comparación Duncan, al 0.05\%. Sistema vertical.

\begin{tabular}{lrc}
\hline T COMPOSICIÓN & PROMEDIO & \\
\hline T7 Suelo virgen + guano fermentado sin microorganismo & 14.729 & A \\
T8 Suelo virgen +guano fermentado con microorganismo 1 & 14.588 & A \\
T9 Suelo virgen +guano fermentado con microorganismo 2 & 14.224 & A \\
T6 Suelo virgen + guano de isla con microorganismo 2 & 5.815 & B \\
T4 Suelo virgen + guano de isla sin microorganismo & 5.623 & B C \\
T5 Suelo virgen + guano de isla con microorganismo 1 & 5.395 & B C \\
T3 Suelo virgen + microorganismo 2 & 3.314 & B C \\
T2 Suelo virgen + microorganismo 1 & 3.018 & B C \\
T1 Suelo virgen sin microorganismo & 2.346 & C \\
\hline
\end{tabular}


Tabla 8. Promedio de peso fresco de hipocótilos en los Tratamientos (T) y comparación Duncan al 0.05\%. Sistema horizontal.

\begin{tabular}{crc}
\hline T COMPOSICIÓN & PROMEDIO & \\
\hline T9 Suelo virgen + guano fermentado con microorganismo 2 & 22.563 & A \\
T7 Suelo virgen + guano fermentado sin microorganismo & 19.791 & A B \\
T8 Suelo virgen + guano fermentado con microorganismo 1 & 15.298 & B C \\
T6 Suelo virgen + guano de isla con microorganismo 2 & 13.685 & C D \\
T5 Suelo virgen + guano de isla con microorganismo 1 & 13.248 & C D \\
T4 Suelo virgen + guano de isla sin microorganismo & 12.784 & C D \\
T3 Suelo virgen + microorganismo 2 & 10.981 & C D \\
T1 Suelo virgen sin microorganismo & 8.955 & D \\
T2 Suelo virgen + microorganismo 1 & 8.346 & D \\
\hline
\end{tabular}

Tabla 9. Análisis de varianza para peso fresco total (g) en los sistemas horizontal y vertical.

\begin{tabular}{lccc}
\hline $\begin{array}{l}\text { Fuente } \\
\text { variación }\end{array}$ & GL & \multicolumn{2}{c}{ Cuadrados Medios } \\
\cline { 3 - 4 } Bloques & 3 & Horizontal & Vertical \\
Tratamientos & 2 & 34.423 & 7.112 \\
Error & 6 & 83.164 & $222.446^{* *}$ \\
Total & 11 & 22.070 & 4.542 \\
C.V. (\%) & & & \\
Promedio & & 28.606 & 19.639 \\
\hline
\end{tabular}

\section{Agradecimientos.}

Deseamos expresar nuestro sincero agradecimiento al Dr. Carlos Samaniego, Director de la empresa ECOANDINO y al CONCYTEC que, a través del FINCYT, se pudo financiar el Proyecto 066-PIPEA2012-FINCYT para esta investigación. Además, reconocemos el apoyo del Ing. MSc. Julián Chura del Programa de maíz - UNALM por los análisis estadísticos realizados.

\section{Literatura citada.}

Alcántara H. 2015. Junín: siembra de maca crea tráfico de tierras. En Diario Correo del 26 de mayo del 2015. Recuperado de http://www.diariocorreo.pe/.

Aliaga R. 1999. Guía para el cultivo, aprovechamiento y conservación de la maca. Convenio Andrés Bello. SECAB. Ciencia y Tecnología no. 82. Santafé de Bogotá.

Black C. 1973. Photosynthetic carbon fixation in relation to net $\mathrm{CO} 2$ uptake. Annual Review of Plant Physiology 24: 253-286.

Calderón A. 1988. Efectos de dos microclimas contrastantes en la composición bioquímica de los tubérculos de diez genotipos de papa. Tesis (Biólogo). Lima, Perú. Universidad Nacional Agraria La Molina, Facultad de Ciencias.

Calvo P. 2008. Capacidad PGPR de bacterias del género Bacillus aisladas de la rizosfera del cultivo de papa (Solanum tuberosum L.) en los andes del Perú. Tesis (Biólogo). Lima, Perú. Universidad Nacional Agraria La Molina, Facultad de Ciencias.

Calzada J. 1970. Métodos estadísticos para la investigación. Editorial Jurídica S.A. Lima, Perú. 156-206.

Dini A., Mglivolo G., Rastrelli L., Saturnino P. \& Sehettiono O. 1994. Chemical composition of Lepidium meyenii Walp. Food chemistry. 49: 347-349.
Fageria N., Baligar V. \& Clark R. 2006. Physiology of crop Production. Food Products. Press. The Haworth Press. Inc. New York. London. Oxford.

Garay O. 1992. Cultivo de la Maca. Instituto Nacional de Investigación Agraria y Agroindustrial. Lima, Perú. Serie divulgativa: Folleto-Instituto Nacional de Investigación Agraria y Agroindustrial (Perú) no. 16.

Heller R., Esnault R. \& Lance C. 2000. Physiologie vègètale. Tome 2. Developpement. èdition de l'Abrégé. Dunod, Paris.

Johns T. 1981. The añu and the maca. Journal of Ethnobiology 1: 208-212.

King S. R. 1988. Economic Botany of the Andean Tuber crop complex Lepidium meyenii, Oxalis tuberosa, Tropaeolum tuberosum and Ullucus tuberosus. Tesis PhD. University of New York, EE UU.

La República. 2017. Crisis en el cultivo y producción de maca por apropiación genética de China. En: La República del 5 de julio del 2017. Recuperado de: https://larepublica.pe/economia/1058354-crisis-en-elcultivo-y-produccion-de-maca-por-apropiaciongenetica-de-china.

Marthe F., Schütze W., Krüger H., Scholze P., Kramer R. \& Ryschka U. 2003. Maca (Lepidium meyenii): cultivation, resistance and composition of secondary metabolites under European conditions. Poster Session. In: Knüpffer H. \& Ochsmann J. (Eds). 2003. Rudolf Mansfeld and Plant Genetic Resources. Proceedings of a symposium dedicated to the 100th birthday of Rudolf Mansfeld, Gatersleben, Germany, 8-9 October 2001. 290 - 293.

Moreno U. 1970. Physiological investigations on the potato plant. Tesis Ph.D. Universidad de Cornell, Ithaca, New York.

Moreno U. 1985. Environmental effects on growth and development of potato plants. Chapter 14. Potato Physiology. Ed. Academic Press Inc. London. Chapter 14: 481-500.

Obregón L. 1998. Maca, planta medicinal y nutritiva del Perú. Instituto de Fitoterapia Americana.

Quiros C., Epperson A., Hu J. \& Holle M. 1996. Physiological Studies and Determination of Chromosome Number in Maca, Lepidium meyenii (Brassicaceae). Economic Botany. N.Y. Botanical Garden, EE UU. 50: 216-223.

Salisbury F. \& Ross C.1994. Fisiología Vegetal. Editorial Ibero América. México.

Strasburger E., Noll F., Schenck H. \& Schimper A.F.W. 1986. Tratado de Botánica. Editorial Marín S.A Barcelona. Séptima Edición Española. 
Taiz L. \& Zeiger E. 2011. Plant Physiology. Fifth Edition. Sinauer Associates Inc. Publishers. Sunderland Massachusetts. EE UU.

Tapia M. 1991. Agronomía de los cultivos andinos subexplotados. Cultivos andinos subexplotados y su aporte a la alimentación. Santiago de Chile FAO. Oficina Regional para América Latina y el Caribe.
Tello J., Herman M. \& Calderón A. 1992. La Maca (Lepidium meyenii Walp) Cultivo alimenticio potencial para las zonas alto andinas. Boletín de Lima. 81: 59-66.

\section{Apéndice.}

Anexo 1a. Datos meteorológicos dentro y fuera del invernadero. Hora $08.00 \mathrm{am}$.

\begin{tabular}{lcccccrrr}
\hline \multirow{2}{*}{ MES } & \multicolumn{2}{c}{ TEMPERATURA AIRE ${ }^{\circ} \mathrm{C}$} & \multicolumn{2}{c}{ HUMEDAD AIRE \% } & \multicolumn{2}{c}{ ILUMINANCIA } \\
\cline { 2 - 8 } & DENTRO & FUERA & DENTRO & FUERA & DENTRO & FUERA & DENTRO & FUERA \\
\hline Octubre & 15.43 & 14.28 & 50.3 & 52.8 & 25258.00 & 31310.76 & 144.50 & 110.33 \\
Noviembre & 19.30 & 21.01 & 39.1 & 36.9 & 28283.68 & 35356.27 & 84.95 & 81.93 \\
Diciembre & 15.44 & 15.78 & 48.9 & 48.7 & 23915.93 & 29212.00 & 42.47 & 40.24 \\
Enero & 14.09 & 14.46 & 54.1 & 52.4 & 20649.80 & 29450.20 & 16.53 & 16.32 \\
Febrero & 15.18 & 17.55 & 52.8 & 44.5 & 23838.29 & 33161.41 & 36.32 \\
Marzo & 13.19 & 15.86 & 56.9 & 48.2 & 24131.68 & 31897.28 & 13.48 & 17.76 \\
Abril & 13.33 & 15.81 & 52.5 & 45.5 & 28142.10 & 32340.95 & 10.83 & 12.38 \\
Mayo & 14.19 & 15.35 & 45.9 & 43.8 & 32373.10 & 31577.48 & 11.03 & 12.00 \\
Junio & 19.98 & 15.68 & 41.7 & 42.6 & 32627.33 & 35406.67 & 10.83 & 13.33 \\
Promedio & 15.24 & 16.19 & 49.14 & 46.15 & 26579.99 & 32190.33 & 41.22 & 36.70 \\
\hline
\end{tabular}

Anexo 1b. Datos meteorológicos dentro fuera del invernadero. Hora $12.00 \mathrm{am}$.

\begin{tabular}{|c|c|c|c|c|c|c|c|c|}
\hline \multirow{2}{*}{ MES } & \multicolumn{2}{|c|}{ TEMPERATURA AIRE ${ }^{\circ} \mathrm{C}$} & \multicolumn{2}{|c|}{ HUMEDAD AIRE \% } & \multicolumn{2}{|c|}{ ILUMINANCIA } & \multicolumn{2}{|c|}{$\mathrm{CO} 2 \mathrm{ppm}$} \\
\hline & DENTRO & FUERA & DENTRO & FUERA & DENTRO & FUERA & DENTRO & FUERA \\
\hline Octubre & 23.85 & 22.00 & 34.4 & 35.3 & 31867.25 & 39020.50 & 119.50 & 111.00 \\
\hline Noviembre & 31.96 & 28.16 & 22.4 & 27.7 & 31992.84 & 36959.13 & 102.16 & 86.87 \\
\hline Diciembre & 25.11 & 25.85 & 33.0 & 33.1 & 31977.68 & 37740.74 & 56.50 & 51.00 \\
\hline Enero & 24.12 & 26.88 & 34.3 & 31.7 & 29561.10 & 39547.87 & 26.43 & 26.53 \\
\hline Febrero & 23.58 & 23.89 & 34.8 & 36.4 & 31342.43 & 36085.79 & 31.68 & 29.68 \\
\hline Marzo & 26.02 & 28.95 & 32.5 & 29.3 & 34604.42 & 40465.87 & 22.03 & 29.91 \\
\hline Abril & 22.23 & 23.63 & 31.6 & 30.6 & 31256.53 & 37653.43 & 17.10 & 22.43 \\
\hline Mayo & 24.53 & 25.77 & 22.2 & 23.1 & 33910.61 & 35999.91 & 15.61 & 16.17 \\
\hline Junio & 28.92 & 27.75 & 20.9 & 20.4 & 33721.00 & 37159.33 & 16.83 & 14.83 \\
\hline Promedio & 25.59 & 25.87 & 29.5 & 29.7 & 32248.21 & 36736.95 & 45.31 & 43.15 \\
\hline
\end{tabular}

Anexo 1c. Datos meteorológicos dentro y fuera del invernadero. . Hora $03.00 \mathrm{pm}$.

\begin{tabular}{|c|c|c|c|c|c|c|c|c|}
\hline \multirow{2}{*}{ MES } & \multicolumn{2}{|c|}{ TEMPERATURA AIRE ${ }^{\circ} \mathrm{C}$} & \multicolumn{2}{|c|}{ HUMEDAD AIRE \% } & \multicolumn{2}{|c|}{ ILUMINANCIA } & \multicolumn{2}{|c|}{$\mathrm{CO} 2 \mathrm{ppm}$} \\
\hline & DENTRO & FUERA & DENTRO & FUERA & DENTRO & FUERA & DENTRO & FUERA \\
\hline Octubre & 22.72 & 19.25 & 31.87 & 35.85 & 26344.33 & 34521.75 & 127.00 & 129.25 \\
\hline Noviembre & 26.17 & 27.16 & 28.26 & 27.15 & $31188 . .72$ & 36472.90 & 95.50 & 80.20 \\
\hline Enero & 21.83 & 23.94 & 36.77 & 35.01 & 40638.00 & 36596.00 & 20.86 & 22.25 \\
\hline Febrero & 21.95 & 22.74 & 39.65 & 37.47 & 26458.82 & 32637.11 & 29.18 & 28.11 \\
\hline Marzo & 19.73 & 22.68 & 38.75 & 35.25 & 26272.43 & 33467.92 & 20.00 & 18.00 \\
\hline Junio & 22.19 & 23.15 & 34.56 & 33.67 & 29634.70 & 36984.78 & 46.46 & 43.12 \\
\hline Promedio & 22.18 & 23.15 & 34.56 & 33.7 & 29634.70 & 36984.70 & 46.47 & 43.00 \\
\hline
\end{tabular}

${ }^{1}$ Departamento Académico de Biología, Universidad Nacional Agraria La Molina, Av. La Molina s/n, Lima-Perú, acalderon@lamolina.edu.pe.

2 Asistente del Laboratorio de Fisiología Vegetal, Departamento Académico de Biología, Universidad Nacional Agraria La Molina, Av. La Molina s/n., Lima-Perú. 